Discussiones Mathematicae

Probability and Statistics 37 (2017) 135-146

doi:10.7151/dmps.1192

\title{
STRUCTURED STAIR NESTING MODELS-TWO APPROACHES
}

\author{
SAndra InÊs DA Cunha Monteiro \\ Dina Maria Morgado Salvador \\ Center of Mathematics and Applications-New University of Lisbon \\ Polytechnic Institute of Setúbal \\ e-mail: sandra.ines.monteiro@esce.ips.pt \\ dina.salvador@estsetubal.ips.pt \\ AND \\ João Tiago Praça Nunes Mexia \\ Center of Mathematics and Applications-New University of Lisbon \\ e-mail: jtm@fct.unl.pt
}

\begin{abstract}
The structured stair nesting model was proposed by [4] and it was introduced according with a step by step approach. The purpose of this work is to show that structured stair nesting models can be analyzed with a diferent approach, which we call global approach. We also show that, the two approaches, although originating different algebraic structures, allow to obtain the same estimators for the variance components. To express the algebraic structure of these model we will use commutative Jordan algebras.
\end{abstract}

Keywords: structured stair nesting models, commutative Jordan algebras, variance components.

2010 Mathematics Subject Classification: 62J12.

\section{INTRODUCTION}

Stair nesting models are a good alternative to the use of models with balanced nesting, see [3], because they allow to analyze the same number of factors with less 
observations. The stair nesting models were introduced by [1] but its algebraic structure was only proposed later by [2].

In order to allow more than one factor on each step, a new type of model was proposed by [4] that was called structured stair nesting model. This model was introduced using an approach that we begin now calling step by step approach. Step by step approach analyses the model by dividing it in several submodels, i.e., instead having only one model there are as many submodels as the number of steps in the inicial model. In [5] were obtained estimators for the components of variance. Structured stair nesting models have allowed the construction of more complex models, since they allow the increase the number of analysed factors, for the same number of treatments. The number of factors, instead of the number of steps, became the sum of the number of factors for the submodels.

The goal of this work is to present structured stair nesting models but with a different approach, which we define as global approach. We now compare the step by step approach with the global one, in which we have an unique model. Both approaches will use commutative Jordan algebras. When we use the step by step approach we call the model as step by step model, otherwise we call the model as global model.

\section{Step By Step Model}

\subsection{Algebraic structure}

Let $u$ be the number of steps, $a(h)$ the number of treatments, in the $h^{\text {th }}$ step and $n=\sum_{h=1}^{u} a(h)$ the total number of treatments. Let $w(h)$ be the number of incidence matrices in the $h^{\text {th }}$ step. Following [5], the random effects structured stair nesting model is given by

$$
\mathbf{y}=\sum_{h=0}^{u} \mathbf{X}(h) \boldsymbol{\beta}(h)
$$

with the incidence matrices $\mathbf{X}(h), h=0, \ldots, u$, defined as

$$
\left\{\begin{array}{l}
\mathbf{X}(0)=D\left(\mathbf{1}_{a(1)}, \mathbf{1}_{a(2)}, \ldots, \mathbf{1}_{a(u)}\right) \\
\mathbf{X}(h)=D\left(\mathbf{I}_{a(1)}, \ldots, \mathbf{I}_{a(h-1)},\left[\dot{\mathbf{X}}_{1}(h), \ldots, \dot{\mathbf{X}}_{w(h)}(h)\right], \mathbf{1}_{a(h+1)}, \ldots, \mathbf{1}_{a(u)}\right)
\end{array}\right.
$$


and the effects vectors $\boldsymbol{\beta}(h), h=0, \ldots, u$, given by

$$
\left\{\begin{array}{l}
\boldsymbol{\beta}(0)=\left[\beta_{1}(0), \ldots, \beta_{u}(0)\right]^{t} \\
\boldsymbol{\beta}(h)=\left[\boldsymbol{\beta}_{1}^{t}(h), \ldots, \boldsymbol{\beta}_{h-1}^{t}(h),\left[\dot{\boldsymbol{\beta}}_{1}^{t}(h), \ldots, \dot{\boldsymbol{\beta}}_{w(h)}^{t}(h)\right]^{t}, \beta_{h+1}(h), \ldots, \beta_{u}(h)\right]^{t} .
\end{array}\right.
$$

We assume that $\boldsymbol{\beta}(0)=\mathbf{1}_{u} \mu$, with $\mu$ the general mean value and that the vectors $\boldsymbol{\beta}(h), h=1, \ldots, u$, are random and independents with null mean vectors and variance covariance matrices $\sigma^{2}(h) \mathbf{I}_{c(h)}, h=1, \ldots, u$, this is,

$$
\boldsymbol{\beta}(h) \sim\left(\mathbf{0}_{c(h)} ; \sigma^{2}(h) \mathbf{I}_{c(h)}\right), h=1, \ldots, u,
$$

where

$$
c(h)=\sum_{l=1}^{h-1} a(l)+\sum_{i=1}^{w(h)} c_{i}(h)+u-h, h=1, \ldots, u,
$$

with

$$
c_{j}(h)=\operatorname{car}\left(\dot{\mathbf{X}}_{j}(h)\right), h=1, \ldots, u, j=1, \ldots, w(h) .
$$

With step by step approach we can rewrite the model (1) considering subvectors, i.e.,

$$
\mathbf{y}=\left[\mathbf{y}^{t}(1) \cdots \mathbf{y}^{t}(u)\right]^{t}
$$

with each subvector

$$
\begin{aligned}
\mathbf{y}(h) & =\beta_{h}(0) \mathbf{1}_{a(h)}+\sum_{\ell=1}^{h-1} \beta_{h}(\ell) \mathbf{1}_{a(h)}+\sum_{j=1}^{w(h)} \dot{\mathbf{X}}_{j}(h) \dot{\boldsymbol{\beta}}_{j}(h) \\
& +\sum_{\ell=h+1}^{u} \mathbf{I}_{a(h)} \boldsymbol{\beta}_{h}(\ell), h=1, \ldots, u
\end{aligned}
$$

being the submodel in the $h^{\text {th }}$ step, $h=1, \ldots, u$.

We assume that $\beta_{1}(0), \ldots, \beta_{u}(0)$ are fixed and the remaining subvectors to be independent and

$$
\begin{cases}\beta_{h}(\ell) \sim\left(0 ; \sigma^{2}(\ell)\right) & h=1, \ldots, u, \quad \ell=1, \ldots, h-1 \\ \boldsymbol{\beta}_{h}(\ell) \sim\left(\mathbf{0}_{a(h)} ; \sigma^{2}(\ell) \mathbf{I}_{a(h)}\right) & h=1, \ldots, u, \quad \ell=h+1, \ldots, u \\ \dot{\boldsymbol{\beta}}_{j}(h) \sim\left(\mathbf{0}_{c_{j}(h)} ; \dot{\sigma}_{j}^{2}(h) \mathbf{I}_{c_{j}(h)}\right) & h=1, \ldots, u, \quad j=1, \ldots, w(h)\end{cases}
$$


where

$$
\sum_{j=1}^{w(h)} \dot{\sigma}_{j}^{2}(h)=\sigma^{2}(h)
$$

The submodels $\mathbf{y}(h), h=1, \ldots, u$, are independent with mean vectors $\boldsymbol{\mu}(h)=\mathbf{1}_{a(h)} \mu, h=1, \ldots, u$ and variance covariance matrices

$$
\mathbf{V}(h)=\sum_{\ell=1}^{h-1} \sigma^{2}(\ell) \mathbf{J}_{a(h)}+\sum_{j=1}^{w(h)} \dot{\sigma}_{j}^{2}(h) \dot{\mathbf{M}}_{j}(h)+\sum_{\ell=h+1}^{u} \sigma^{2}(\ell) \mathbf{I}_{a(h)}, h=1, \ldots, u,
$$

where

$$
\dot{\mathbf{M}}_{j}(h)=\dot{\mathbf{X}}_{j}(h) \dot{\mathbf{X}}_{j}^{t}(h), h=1, \ldots, u, j=1, \ldots, w(h)
$$

Assuming that, in $h^{\text {th }}$ step, $h=1, \ldots, u$, the matrices (9) commute, then the family

$$
\underline{\dot{M}}(h)=\left\{\dot{\mathbf{M}}_{1}(h), \ldots, \dot{\mathbf{M}}_{w(h)}(h)\right\}, h=1, \ldots, u
$$

generates a commutative Jordan algebra $\dot{\mathcal{A}}(a(h)), h=1, \ldots, u$, with principal basis

$$
\underline{\dot{Q}}(h)=b p(\dot{\mathcal{A}}(a(h)))=\left\{\dot{\mathbf{Q}}_{1}(h), \ldots, \dot{\mathbf{Q}}_{m(h)}(h)\right\}, h=1, \ldots, u,
$$

and

$$
\dot{\mathbf{M}}_{j}(h)=\sum_{k=1}^{m(h)} b_{j, k}(h) \dot{\mathbf{Q}}_{k}(h), j=1, \ldots, w(h), h=1, \ldots, u .
$$

Then

$$
\mathbf{B}(h)=\left[b_{j, k}(h)\right], h=1, \ldots, u, j=1, \ldots, w(h), k=1, \ldots, m(h)
$$

is designated as the $\underline{\dot{M}}(h) \backslash \underline{\dot{Q}}(h)$ transition matrix.

We also have the square roots

$$
[\underline{\dot{Q}}(h)]^{\frac{1}{2}}=b p^{\frac{1}{2}}(\dot{\mathcal{A}}(a(h)))=\left\{\dot{\mathbf{A}}_{1}(h), \ldots, \dot{\mathbf{A}}_{m(h)}(h)\right\},
$$

with

$$
\dot{\mathbf{Q}}_{k}(h)=\dot{\mathbf{A}}_{k}^{t}(h) \dot{\mathbf{A}}_{k}(h), h=1, \ldots, u, k=1, \ldots, m(h)
$$


and

$$
\dot{\mathbf{A}}_{1}(h)=\frac{1}{\sqrt{a(h)}} \mathbf{1}_{a(h)}^{t}, h=1, \ldots, u .
$$

Using (12), we can write

$$
\mathbf{V}(h)=\sum_{k=1}^{m(h)} \dot{\gamma}_{k}(h) \dot{\mathbf{Q}}_{k}(h), h=1, \ldots, u,
$$

with

$$
\left\{\begin{array}{l}
\dot{\gamma}_{1}(h)=a(h) \sum_{\ell=1}^{h-1} \sigma^{2}(\ell)+\sum_{j=1}^{w(h)} \dot{\sigma}_{j}^{2}(h) b_{j, 1}(h)+\sum_{\ell=h+1}^{u} \sigma^{2}(\ell) \\
\dot{\gamma}_{k}(h)=\gamma_{k}(h)+\sum_{\ell=h+1}^{u} \sigma^{2}(\ell) \\
\gamma_{k}(h)=\sum_{j=1}^{w(h)} \dot{\sigma}_{j}^{2}(h) b_{j, k}(h) .
\end{array}\right.
$$

\subsection{Variance components}

Considering the $\underline{\dot{M}}(h) \backslash \underline{\dot{Q}}(h)$ transition matrix

$$
\mathbf{B}(h)=\left[\begin{array}{cc}
b_{11}(h) & \mathbf{0}^{t} \\
\mathbf{b}_{21}(h) & \mathbf{B}_{22}(h)
\end{array}\right], h=1, \ldots, u,
$$

and the vectors of the variance componentes

$$
\left\{\begin{array}{c}
\boldsymbol{\sigma}_{\mathbf{2}}^{2}(h)=\left[\dot{\sigma}_{2}^{2}(h) \cdots \dot{\sigma}_{w(h)}^{2}(h)\right]^{t}, \quad h=1, \ldots, u \\
\dot{\gamma}_{\mathbf{2}}(h)=\left[\dot{\gamma}_{2}(h) \cdots \dot{\gamma}_{m(h)}(h)\right]^{t}, \quad h=1, \ldots, u
\end{array}\right.
$$

then, if $\mathbf{B}_{22}(h)$ has linearly independent rows,

$$
\boldsymbol{\sigma}_{\mathbf{2}}^{\mathbf{2}}(h)=\left(\mathbf{B}_{22}^{t}(h)\right)^{+}\left(\dot{\gamma}_{\mathbf{2}}(h)-\left(\sum_{\ell=h+1}^{u} \sigma^{2}(\ell)\right) \mathbf{1}_{(m(h)-1)}\right), h=1, \ldots, u,
$$

with ${ }^{+}$indicating Moore Penrose inverse, and

$$
\sigma^{2}(\ell)=\mathbf{1}^{t} \boldsymbol{\sigma}_{\mathbf{2}}^{\mathbf{2}}(\ell), h=1, \ldots, u, \ell=h+1, \ldots, u .
$$




\subsection{Estimation}

To be able to estimate the initial variance components it is necessary first to estimate the canonical variance components.

Given the vectors

$$
\tilde{\boldsymbol{\eta}}_{k}(h)=\dot{\mathbf{A}}_{k}(h) \mathbf{y}(h), h=1, \ldots, u, k=1, \ldots, m(h)
$$

we have that

$$
\begin{cases}\dot{\boldsymbol{\eta}}_{k}(h) \sim\left(\dot{\boldsymbol{\eta}}_{k}(h) ; \dot{\gamma}_{k}(h) \mathbf{I}_{g_{k}(h)}\right), & h=1, \ldots, u, \quad k=1, \ldots, m(h) \\ \dot{\boldsymbol{\eta}}_{k}(h)=\dot{\mathbf{A}}_{k}(h) \boldsymbol{\mu}(h), & h=1, \ldots, u, \quad k=1, \ldots, m(h) \\ g_{k}(h)=\operatorname{car}\left(\dot{\mathbf{A}}_{k}(h)\right), & h=1, \ldots, u, \quad k=1, \ldots, m(h) .\end{cases}
$$

As

$$
\tilde{\dot{\boldsymbol{\eta}}}_{k}(h) \sim\left(\mathbf{0}_{g_{k}(h)} ; \dot{\gamma}_{k}(h) \mathbf{I}_{g_{k}(h)}\right), h=1, \ldots, u, k=2, \ldots, m(h),
$$

then we get the unbiased estimators

$$
\tilde{\dot{\gamma}}_{k}(h)=\frac{\left\|\dot{\mathbf{A}}_{k}(h) \mathbf{y}(h)\right\|^{2}}{g_{k}(h)}, h=1, \ldots, u, k=2, \ldots, m(h) .
$$

\section{Global Model}

\subsection{Algebraic structure}

In order to apply the global approach to structured stair nesting models we need to redefine the matrices (2) and the vectors (3). These changes allow the structured stair nesting model to be treated like a single model. However, as we shall see, we obtain the same estimators for the variance components.

The random effects structured stair nesting model is given by

$$
\mathbf{y}=\sum_{h=0}^{u} \mathbf{X}(h) \boldsymbol{\beta}(h)
$$

with

$$
\left\{\begin{array}{l}
\mathbf{X}(0)=D\left(\mathbf{1}_{a(1)}, \mathbf{1}_{a(2)}, \ldots, \mathbf{1}_{a(u)}\right) \\
\mathbf{X}(h)=\left[\mathbf{X}_{1}(h) \ldots \mathbf{X}_{w(h)}(h)\right], \quad h=1, \ldots, u
\end{array}\right.
$$


where, to $h=1, \ldots, u, i=1, \ldots, w(h)$,

$$
\mathbf{X}_{i}(h)=D\left(\mathbf{I}_{a(1)}, \ldots, \mathbf{I}_{a(h-1)}, \dot{\mathbf{X}}_{i}(h), \mathbf{1}_{a(h+1)}, \ldots, \mathbf{1}_{a(u)}\right),
$$

and

(30)

$$
\begin{cases}\boldsymbol{\beta}(0)=\left[\beta_{1}(0), \ldots, \beta_{u}(0)\right]^{t} & \\ \boldsymbol{\beta}(h)=\left[\boldsymbol{\beta}_{1}^{t}(h), \ldots, \boldsymbol{\beta}_{w(h)}^{t}(h)\right]^{t}, & h=1, \ldots, u, \quad i=1, \ldots, w(h) \\ \boldsymbol{\beta}_{i}(h)=\left[\mathbf{0}_{a(1)}^{t}, \ldots, \mathbf{0}_{a(h-1)}^{t}, \dot{\boldsymbol{\beta}}_{i}^{t}(h), 0, \ldots, 0\right], & h=1, \ldots, u, \quad i=1, \ldots, w(h) .\end{cases}
$$

Note that the model (27) can still be written as

$$
\mathbf{y}=\boldsymbol{\mu}+\sum_{h=1}^{u} \sum_{i=1}^{w(h)} \mathbf{X}_{i}(h) \boldsymbol{\beta}_{i}(h)
$$

Assuming

$$
\dot{\boldsymbol{\beta}}_{i}(h) \sim\left(\mathbf{0}_{c_{i}(h)} ; \dot{\sigma}_{i}^{2}(h) \mathbf{I}_{c_{i}(h)}\right), h=1, \ldots, u, i=1, \ldots, w(h)
$$

with

$$
c_{i}(h)=\operatorname{car}\left(\dot{\mathbf{X}}_{i}(h)\right)
$$

then

(34) $\operatorname{COV}\left[\boldsymbol{\beta}_{i}(h)\right]=D\left(\mathbf{0}_{a(1) \times a(1)}, \ldots, \mathbf{0}_{a(h-1) \times a(h-1)}, \dot{\sigma}_{i}^{2}(h) \mathbf{I}_{c_{i}(h)}, 0, \ldots, 0\right)$.

The model (31) has mean vector

$$
\boldsymbol{\mu}=\mathbf{1}_{n} \mu
$$

and variance covariance matrix

$$
\mathbf{V}=\sum_{h=1}^{u} \sum_{i=1}^{w(h)} D\left(\mathbf{0}_{a(1) \times a(1)}, \ldots, \dot{\sigma}_{i}^{2}(h) \dot{\mathbf{M}}_{i}(h), \ldots, \mathbf{0}_{a(u) \times a(u)}\right),
$$

where

$$
\dot{\mathbf{M}}_{i}(h)=\dot{\mathbf{X}}_{i}(h) \dot{\mathbf{X}}_{i}^{t}(h), h=1, \ldots, u, i=1, \ldots, w(h)
$$


As in the previous approach, we assume that the matrices (37) commute and so

$$
\dot{\mathbf{M}}_{i}(h)=\sum_{k=1}^{m(h)} b_{i, k}(h) \dot{\mathbf{Q}}_{k}(h), h=1, \ldots, u, i=1, \ldots, w(h)
$$

with $\dot{\mathbf{Q}}_{k}(h), h=1, \ldots, u$, the matrices that belong to the principal basis

$$
\underline{\dot{Q}}(h), h=1, \ldots, u
$$

defined in (11).

The model (31) is associated with the commutative Jordan algebra, $\mathcal{A}$, with principal basis

$$
\underline{Q}=\underset{h=1}{\stackrel{u}{\times}} \dot{Q}(h),
$$

where $X$ represents the cartesian product of principal basis of commutative Jordan algebras. The matrices in $\underline{Q}$ are the block diagonal matrices

$\mathbf{Q}_{k}(h)=D\left(\mathbf{0}_{a(1) \times a(1)}, \ldots, \mathbf{0}_{a(h-1) \times a(h-1)}, \dot{\mathbf{Q}}_{k}(h), \mathbf{0}_{a(h+1) \times a(h+1)}, \ldots, \mathbf{0}_{a(u) \times a(u)}\right)$

where $\dot{\mathbf{Q}}_{k}(h) \in \underline{\dot{Q}}(h), h=1, \ldots, u$.

We also have

$$
\underline{Q}^{\frac{1}{2}}=\underset{h=1}{u}[\underline{\dot{Q}}(h)]^{\frac{1}{2}}=\left\{\mathbf{A}_{k}(h): h=1, \ldots, u, k=1, \ldots, m(h)\right\}
$$

where

$$
\mathbf{A}_{1}(h)=\left[\mathbf{0}_{a(1)}^{t}, \ldots, \mathbf{0}_{a(h-1)}^{t}, \dot{\mathbf{A}}_{1}(h), \mathbf{0}_{a(h+1)}^{t}, \ldots, \mathbf{0}_{a(u)}^{t}\right], h=1, \ldots, u
$$

and

$$
\mathbf{A}_{k}(h)=\left[\mathbf{0}_{g_{k}(h) \times a(1)}, \ldots, \mathbf{0}_{g_{k}(h) \times a(h-1)}, \dot{\mathbf{A}}_{k}(h), \mathbf{0}_{g_{k}(h) \times a(h+1)}, \ldots, \mathbf{0}_{g_{k}(h) \times a(u)}\right],
$$

with

$$
g_{k}(h)=\operatorname{car}\left(\dot{\mathbf{A}}_{k}(h)\right), h=1, \ldots, u, k=2, \ldots, m(h) .
$$


Using (38) we get

(46) $\quad \mathbf{V}=\sum_{h=1}^{u} \sum_{i=1}^{w(h)} \sum_{k=1}^{m(h)} \dot{\sigma}_{i}^{2}(h) b_{i, k}(h) D\left(\mathbf{0}_{a(1) \times a(1)}, \ldots, \dot{\mathbf{Q}}_{k}(h), \ldots, \mathbf{0}_{a(u) \times a(u)}\right)$

and by (41) we have

$$
\mathbf{V}=\sum_{h=1}^{u} \sum_{k=1}^{m(h)} \alpha_{k}(h) \mathbf{Q}_{k}(h)
$$

where

$$
\alpha_{k}(h)=\sum_{i=1}^{w(h)} \dot{\sigma}_{i}^{2}(h) b_{i, k}(h), h=1, \ldots, u, k=1, \ldots, m(h)
$$

represents the relationship between the canonical components and the initial ones.

\subsection{Variance components}

Consider

$$
\left\{\begin{array}{c}
\boldsymbol{\alpha}=\left[\begin{array}{c}
\boldsymbol{\alpha}(1) \\
\vdots \\
\boldsymbol{\alpha}(u)
\end{array}\right] \quad \text { with } \quad \boldsymbol{\alpha}(h)=\left[\begin{array}{c}
\alpha_{1}(h) \\
\vdots \\
\alpha_{m(h)}(h)
\end{array}\right], \quad h=1, \ldots, u \\
\boldsymbol{\sigma}^{\mathbf{2}}=\left[\begin{array}{c}
\boldsymbol{\sigma}^{\mathbf{2}}(1) \\
\vdots \\
\boldsymbol{\sigma}^{\mathbf{2}}(u)
\end{array}\right] \quad \text { with } \boldsymbol{\sigma}^{\mathbf{2}}(h)=\left[\begin{array}{c}
\dot{\sigma}_{1}^{2}(h) \\
\vdots \\
\dot{\sigma}_{w(h)}^{2}(h)
\end{array}\right], h=1, \ldots, u
\end{array}\right.
$$

and the transition matrix

$$
\mathbf{B}=\left[\mathbf{B}_{i j}\right]=\left[\begin{array}{cccc}
\mathbf{B}_{11} & \mathbf{B}_{12} & \ldots & \mathbf{B}_{1 u} \\
\mathbf{B}_{21} & \mathbf{B}_{22} & \ldots & \mathbf{B}_{2 u} \\
\vdots & & & \\
\mathbf{B}_{u 1} & \mathbf{B}_{u 2} & \ldots & \mathbf{B}_{u u}
\end{array}\right]
$$

where

$$
\mathbf{B}_{i, j}= \begin{cases}a(j) \mathbf{1}_{w(i)} \boldsymbol{\delta}_{m(j)}^{t}, & i<j \\ \mathbf{B}(i), & i=j \\ \mathbf{1}_{w(i)} \mathbf{1}_{m(j)}^{t}=\mathbf{J}_{w(i) \times m(j)}, & i>j\end{cases}
$$


Then we can write

$$
\boldsymbol{\alpha}=\mathbf{B}^{t} \boldsymbol{\sigma}^{\mathbf{2}} \Leftrightarrow \boldsymbol{\alpha}(h)=\sum_{\ell=1}^{u}\left[\mathbf{B}_{\ell h}\right]^{t} \boldsymbol{\sigma}^{2}(\ell), h=1, \ldots, u .
$$

Consider

$$
\boldsymbol{\alpha}(h)=\left[\begin{array}{l}
\boldsymbol{\alpha}_{1}(h) \\
\boldsymbol{\alpha}_{\mathbf{2}}(h)
\end{array}\right]
$$

with

$$
\left\{\begin{array}{cc}
\boldsymbol{\alpha}_{\mathbf{1}}(h)=\left[\alpha_{1}(h)\right], & h=1, \ldots, u \\
\boldsymbol{\alpha}_{\mathbf{2}}(h)=\left[\begin{array}{c}
\alpha_{2}(h) \\
\vdots \\
\alpha_{m(h)}(h)
\end{array}\right], & h=1, \ldots, u
\end{array}\right.
$$

and

$$
\boldsymbol{\sigma}^{2}(h)=\left[\begin{array}{l}
\sigma_{1}^{2}(h) \\
\sigma_{2}^{2}(h)
\end{array}\right]
$$

with

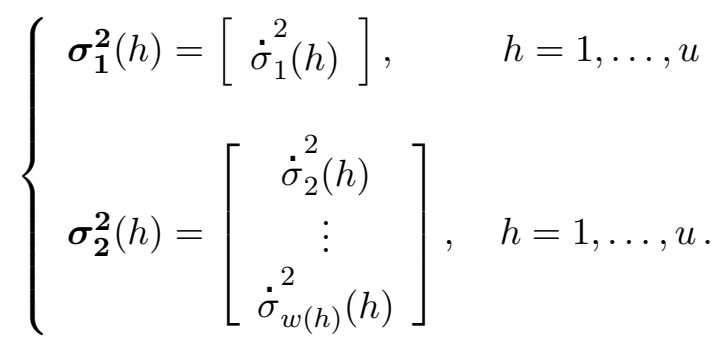

If we consider that the matrix

$$
\mathbf{B}_{i j}^{*}, i, j=1, \ldots, u
$$

is obtained by removing the $1^{\text {st }}$ row and the $1^{\text {st }}$ column of the matrix $\mathbf{B}_{i j}$, we can write

$$
\boldsymbol{\alpha}_{\mathbf{2}}(h)=\sum_{\ell=1}^{u}\left[\mathbf{B}_{\ell h}^{*}\right]^{t} \boldsymbol{\sigma}_{\mathbf{2}}^{\mathbf{2}}(\ell), h=1, \ldots, u .
$$

By (51), we have

$$
\boldsymbol{\alpha}_{\mathbf{2}}(h)=\left[\mathbf{B}^{*}(h)\right]^{t} \boldsymbol{\sigma}_{\mathbf{2}}^{\mathbf{2}}(h)+\sum_{\ell=h+1}^{u} \sigma^{2}(\ell) \mathbf{1}_{m(h)-1}, h=1, \ldots, u .
$$


If the matrices $\left[\mathbf{B}^{*}(h)\right], h=1, \ldots, u$, have linearly independent row vectors then

$$
\boldsymbol{\sigma}_{\mathbf{2}}^{\mathbf{2}}(h)=\left(\left[\mathbf{B}^{*}(h)\right]^{t}\right)^{+}\left[\boldsymbol{\alpha}_{\mathbf{2}}(h)-\sum_{\ell=h+1}^{u} \sigma^{2}(\ell) \mathbf{1}_{m(h)-1}\right], h=1, \ldots, u
$$

and

$$
\sigma^{2}(\ell)=\sum_{i=2}^{w(\ell)} \dot{\sigma}_{i}^{2}(\ell)=\mathbf{1}_{w(\ell)-1}^{t} \boldsymbol{\sigma}_{\mathbf{2}}^{\mathbf{2}}(\ell), \ell=h+1, \ldots, u .
$$

The relationship between the canonical components and the initial ones in this approach is the same that was obtained with the other approach.

\subsection{Estimation}

Let us now see that, although both models in the two approaches are different, we obtain for both the same estimators for the canonical variance components.

Given the vectors

$$
\widetilde{\boldsymbol{\eta}}_{k}(h)=\mathbf{A}_{k}(h) \mathbf{y}, h=1, \ldots, u, k=1, \ldots, m(h)
$$

we have

$$
\left\{\begin{array}{lll}
\widetilde{\boldsymbol{\eta}}_{k}(h) \sim\left(\boldsymbol{\eta}_{k}(h) ; \alpha_{k}(h) \mathbf{I}_{g_{k}(h)}\right), & h=1, \ldots, u, & k=1, \ldots, m(h) \\
\boldsymbol{\eta}_{k}(h)=\mathbf{A}_{k}(h) \boldsymbol{\mu}, & h=1, \ldots, u, & k=1, \ldots, m(h) \\
g_{k}(h)=\operatorname{car}\left(\mathbf{A}_{k}(h)\right), & h=1, \ldots, u, & k=2, \ldots, m(h) .
\end{array}\right.
$$

Since

$$
\widetilde{\boldsymbol{\eta}}_{k}(h) \sim\left(\mathbf{0}_{g_{k}(h)} ; \alpha_{k}(h) \mathbf{I}_{g_{k}(h)}\right), h=1, \ldots, u, k=2, \ldots, m(h)
$$

we get the unbiased estimators

$$
\widetilde{\alpha}_{k}(h)=\frac{\left\|\mathbf{A}_{k}(h) \mathbf{y}\right\|^{2}}{g_{k}(h)}, h=1, \ldots, u, k=2, \ldots, m(h) .
$$

Therefore, the $\widetilde{\alpha}_{k}(h), h=1, \ldots, u, k=2, \ldots, m(h)$, are unbiased estimators to $\alpha_{k}(h), h=1, \ldots, u, k=2, \ldots, m(h)$, through which are obtained estimators to $\dot{\sigma}_{j}^{2}(h)$ and $\sigma^{2}(h), h=1, \ldots, u, j=1, \ldots, w(h)$. 
Given that

$$
\dot{\mathbf{A}}_{k}(h) \mathbf{y}(h)=\mathbf{A}_{k}(h) \mathbf{y}, h=1, \ldots, u, k=2, \ldots, m(h)
$$

this formulation leads to the same statistics than the previous one, which uses the step by step model.

\section{FINAL REMARKS}

In this work we studied structured stair nesting model that allows to study more than one factor by step. We started by presenting some known results of structured stair nesting models using the approach step by step. Next we introduced the global approach to analyze this type of models. Besides presenting its algebraic structure we also obtained estimators for its variance components. The relationship between the initial variance components and canonical ones is equal in the two approaches and the same goes for the estimators of the canonical components. Thus we can conclude that the two approaches give the same estimators for the variance components.

\section{REFERENCES}

[1] D. Cox and P. Solomon, Components of Variance (New York, Chapman and Hall, 2003).

[2] C. Fernandes, P. Ramos and J.T. Mexia, Algebraic structure of step nesting designs, Discuss. Math. Prob. and Statist. 30 (2010) 221-235.

[3] C. Fernandes, P. Ramos and J.T. Mexia, Crossing balance and stair nested design, Electronic J. Linear Algebra, A publication of the International Linear Algebra Society 25 (2012) 22-47.

[4] S. Monteiro, D. Salvador and J.T. Mexia, Advances in structured stair nesting, in: Proceedings of the International Conference on Numerical Analysis and Applied Mathematics ICNAAM, AIP Conf. Proc. 1558 (2013) 851-854.

[5] S. Monteiro, M. Fonseca and F. Carvalho, Estimators for variance components in structured stair nesting models, in: Proceedings of the International Conference on Numerical Analysis and Applied Mathematics ICNAAM, AIP Conf. Proc. 1738 (2016) 060007.

Received 6 May 2017

Revised 19 September 2017 Accepted 28 October 2017 